\title{
Influence of pH and Compression on Electrohydrodynamic Effects in Nanoporous Packed Beds
}

\author{
Bastian Schaefer and Hermann Nirschl \\ Institut für Mechanische Verfahrenstechnik und Mechanik, Universität Karlsruhe (TH), Straße am Forum 8, \\ 76131 Karlsruhe, Germany \\ Correspondence should be addressed to Bastian Schaefer, bastian.schaefer@mvm.uka.de
}

Received 18 August 2008; Revised 9 January 2009; Accepted 28 January 2009

Recommended by Eiji Iritani

Fluid flow and charge transport in fine structures can be driven both by pressure gradients and by electric fields if electrochemical double layers are present on the surfaces. The interrelated electrohydrodynamic effects may be used to drive liquids without moving parts, for example, in dewatering or in electroosmotic chromatography, or to generate small electric currents. While the electrohydrodynamic transport is well understood for simple geometries, models for porous structures are complex. Furthermore, the interconnected porous structure of a packed bed itself strongly depends on the electrochemical double layers. In this study, the electrohydrodynamic transport in packed beds consisting of boehmite particles with an average diameter of $38 \mathrm{~nm}$ is investigated. We describe a new approach to the electrokinetic effects by treating the packed beds as theoretical sets of cylindrical capillaries. The charge transport and the electrically driven fluid flow predicted with this model agree well with experimental results. Furthermore, the hydraulic permeability was found to be a nonlinear function of the porosity, independent of whether the porosity change is caused by changing the compression or the electrochemical double layer.

Copyright (C) 2009 B. Schaefer and H. Nirschl. This is an open access article distributed under the Creative Commons Attribution License, which permits unrestricted use, distribution, and reproduction in any medium, provided the original work is properly cited.

\section{Introduction}

The packed beds investigated in this study are porous structures consisting of randomly distributed, individual particles compressed between two membranes in a round tube. An increasing compression reduces the size of the pores between the particles and thus the porosity. Generally, an electric current is driven by an electric gradient and a fluid flow is driven by a hydraulic gradient. However, in confined geometries with charged surfaces, hydraulic and electric effects are interrelated since the driving gradient shears off the diffuse part of the electrochemical double layer (EDL) on the surface. If the EDL is sheared off by a pressure-driven flow, the dragged ions constitute a streaming current. Likewise, if the ions are accelerated by an electric field, they drag the adjacent water molecules along and the electroosmotic flow results. This interrelation of fluid flow and charge transport thus depends on the surface charge of the particles, the ion distribution in the EDL, and on the pore structure of the packed bed [1-3].
While relations for this electrohydrodynamic transport (EHT) are well established for simple geometries, examining EHT in a packed bed is complicated because of the complex pore structure. Further, the pore structure itself also depends on the same parameters as the surface charge and the ion distribution in the EDL, that is, the $\mathrm{pH}$ value and the ionic strength of the liquid $[1,4]$. Many researchers describe EHT with elaborate spherical cell models, for which the continuity and stokes equation are solved (see [5] for a recent review). Others solve the Poisson-Boltzmann, continuity, and Stokes equations for different pore geometries assuming negligible surface conductivity [6] or apply molecular dynamics simulations [7]. Due to their complexity, these models are not suitable for practical applications. In this study, we propose a new method for analyzing the EHT. The packed beds are considered as theoretical sets of cylindrical capillaries with an experimentally determined geometry.

The EHT in packed beds is an interesting field of research, that also has commercial applications; packed beds are permeated in many processes such as filtration or 
solid phase extraction, but the high-flow resistance limits the hydraulic permeation if the particles are small $[8,9]$. Electric fields can help to increase the flow $[1,10]$. Likewise, electroosmotic micropumps with porous substrates can drive liquids without needing any moving parts, for example, on microfluidic chips $[11,12]$. The results are also relevant for understanding separator membranes in fuel cells and lithium-ion batteries $[13,14]$.

\section{Theoretical Background}

Fluid flow and charge transport in nanoporous packed beds interact with each other giving reason to the term EHT. The interrelation is caused by the excess of mobile counter ions in the EDL, a diffuse cloud of ions attracted by the particles' surface charge. The surface charge depends on the $\mathrm{pH}$ value of the suspension because it originates from the dissociation reactions of the particles surface groups. It is positive at low $\mathrm{pH}$ values and negative at high $\mathrm{pH}$ values. The $\mathrm{pH}$ value where the surface charge is zero is called isoelectric point (IEP). A detailed description of the origin and the structure of the EDL can be found in [1]. The particle charge is often characterized by the zeta potential $\zeta$, which is the potential difference at the shear plane when the EDL is partly sheared off by a relative motion between the particle and the surrounding liquid. The zeta potential is proportional to the cross coefficients $\left(C_{12}\right.$ and $C_{21}$ in (1) and (2)), which correlate the volumetric flow $\dot{V}$ and the electric current $I$ to the driving gradients of pressure $p$ and electric potential $\Psi$. The exponential decay length of the ion concentration in the diffuse part of the EDL, the Debye length $\kappa^{-1}$, is inversely proportional to the square root of the ionic strength. In this study, with a Debye length $\kappa^{-1}$ of about $3 \mathrm{~nm}$ (at an ionic strength of $0.01 \mathrm{~mol} / \mathrm{L}$ and a temperature of $288 \mathrm{~K}$ ) and an average particle diameter $a$ of about $38 \mathrm{~nm}$, Smoluchowski's assumption of a thin EDL ( $\kappa a \gg 1$ ) is valid. Accordingly, the potential gradients may be considered as one-dimensional, so that the EHT can be expressed as

$$
\begin{gathered}
\dot{V}=\frac{A}{L} \cdot\left(-C_{11} \cdot \Delta p-C_{12} \cdot \Delta \Psi\right), \\
I=\frac{A}{L} \cdot\left(-C_{21} \cdot \Delta p-C_{22} \cdot \Delta \Psi\right),
\end{gathered}
$$

with the thickness of the packed bed $L$ and the cross-section area of the packed bed $A[2,15]$.

The coefficients $C_{11}$ to $C_{22}$ depend on the particle charge and on the pore structure of the packed bed, which is influenced by the formation of agglomerates in the suspension from which the packed bed was formed. The stability of suspensions against agglomeration is described by the DLVO theory $[1,4,8]$. A packed bed formed from a stable suspension has a dense structure with a homogeneous pore size distribution and a low permeability. In contrast, an agglomerated suspension results in a loosely textured packed bed with an inhomogeneous pore size distribution. The large pores between the agglomerates are accountable for the high permeability $[1,16]$.
The pore size also depends on the compression of the packed bed. Increasing the compression mainly reduces the size of the large pores between the agglomerates $[16,17]$. Additionally, when the packed bed is permeated, a drag force acts on every particle; the contact force between the particles increases along the direction of the flow since each particle rests on top of another. Consequently, the contact force at the downstream side equals the contact force at the upstream side plus the pressure force that drives the flow [17], but this effect is neglected in this study as the total compression is much higher than the pressure difference which drives the liquid. Assuming a homogeneous compression, the porosity $\Phi$ is determined from the mass $m_{S}$ and the density $\rho_{S}$ of the particles:

$$
\Phi=\frac{\left(L \cdot A-m_{S} / \rho_{S}\right)}{L \cdot A} .
$$

The porosity has a strong influence on the EHT. The following paragraph discusses the meaning and the physical properties of the four coefficients $C_{11}$ to $C_{22}$.

(i) The flow rate $\dot{V}$ of a liquid driven by the pressure difference $\Delta p$ depends on the cross section area $A$ and the thickness $L$ of the packed bed [18]

$$
\dot{V}=-C_{11} \cdot \frac{A}{L} \cdot \Delta p .
$$

The coefficient $C_{11}$ is the hydraulic permeability as originally defined by Darcy [18]. It has been largely investigated for macroporous packed beds with a homogeneous pore size; briefly, a smaller pore size, as resulting from a smaller particle size or from a lower porosity, causes a lower permeability $[19,20]$. Today, the permeability of a packed bed $K_{\text {Darcy }}$ is usually defined as the product of $C_{11}$ and the viscosity of the liquid $\eta$ [21], but this seems to be problematic when dealing with charged particles, as is shown in the following.

(ii) Electroosmotic flow is driven by an externally applied electric potential difference $\Delta \Psi$ if the counter-ions in the EDL of the particles are accelerated by the electric field and drag the nearby water molecules along $[2,3]$ :

$$
\dot{V}=-C_{12} \cdot \frac{A}{L} \cdot \Delta \Psi .
$$

Since electroosmosis is well understood for cylindrical capillaries, we propose to consider the pores of the packed bed as a theoretical set of cylindrical capillaries with the length $L_{\text {Cap }}$, and the total cross-section area of all capillaries $A_{\text {Cap }}[2]$ :

$$
\dot{V}=-C_{12, \text { Cap }} \cdot \frac{A_{\text {Cap }}}{L_{\text {Cap }}} \cdot \Delta \Psi .
$$

The electroosmosis coefficient $C_{12 \text {,Cap }}$ includes the relative permittivity of the liquid $\varepsilon_{L}$ and the vacuum permittivity $\varepsilon_{0}$ :

$$
C_{12, \text { Cap }}=-\frac{\varepsilon_{L} \varepsilon_{0} \zeta}{\eta} .
$$

The relation between $A_{\text {Cap }} / L_{\text {Cap }}$ and $A / L$ is addressed in the paragraph on the electric conductivity $C_{22}$. 
(iii) The streaming current $I_{\mathrm{Str}}$ is the charge transport created when counter-ions are dragged from the EDL by a pressure-driven liquid flow. The streaming current coefficient $C_{21}$ equals the electroosmosis coefficient $C_{12}$ because of Onsager's relation $[2,3]$. As above, we propose to treat the porous structure as a theoretical set of cylindrical capillaries to make the EHT easier to understand:

$$
\begin{aligned}
& I_{\mathrm{Str}}=-C_{21} \cdot \frac{A}{L} \cdot \Delta p, \\
& I_{\mathrm{Str}}=-C_{21, \text { Cap }} \cdot \frac{A_{\text {Cap }}}{L_{\text {Cap }}} \cdot \Delta p,
\end{aligned}
$$

with the streaming current coefficient $C_{21, \text { Cap }}$. The streaming current could be measured if the inlet area of the structure was connected to the outlet area with a low-resistance ampere meter (short-circuit conditions) [2]. However, it is not investigated in this study since collecting the ions on the large cross-section area of the packed bed with point electrodes is inaccurate.

If the faces are not connected or connected with a highresistance voltage meter, the streaming current is balanced by a charge transport against the pressure-driven flow, the socalled counter current. An electric potential, the streaming potential $\Delta \Psi_{\text {Str }}$, builds up to drive the counter current against the electric resistance of the packed bed. The streaming potential may be calculated from (2) if the electric current $I$ is set to zero [2]. The streaming potential can also be expressed in terms of the capillary geometry with the streaming current coefficient for a capillary and the capillary conductivity $C_{22, \text { Cap }}$ as introduced in the following:

$$
\begin{aligned}
& \Delta \Psi_{\text {Str }}=-\frac{C_{21}}{C_{22}} \cdot \Delta p, \\
& \Delta \Psi_{\text {Str }}=-\frac{C_{21, \text { Cap }}}{C_{22, \text { Cap }}} \cdot \Delta p .
\end{aligned}
$$

Measuring the streaming potential between an upstream and a downstream electrode allows the determination of the zeta potential $\zeta$. It is important to make a correction for surface conduction (see what follows), since it reduces the potential needed to induce the counter current $[1,2]$.

The streaming potential causes an electroosmotic backflow against the pressure gradient, as can be seen when $\Delta \Psi_{\text {Str }}$ is inserted into (1):

$$
\dot{V}_{\text {reduced }}=-\left(C_{11}-\frac{C_{12} \cdot C_{21}}{C_{22}}\right) \cdot \frac{A}{L} \Delta p .
$$

This so-called electroviscous effect appears as an increased apparent viscosity $\eta^{*}$ of the liquid. Imagine a packed bed that is hydraulically permeated while the faces are short circuited, so that no streaming potential and no electroosmotic backflow evolve. If the short circuiting is now cut, the flow rate is reduced by the electroviscosity ratio

$$
\frac{\eta^{*}}{\eta}=\frac{C_{11}}{C_{11}-\left(C_{12} \cdot C_{21} / C_{22}\right)} .
$$

(iv) The electric current $I$ driven by the electric potential difference $\Delta \Psi$ depends on the electric conductivity $C_{22}$ of the porous structure, which is inversely proportional to the ohmic resistance $R_{\mathrm{Ohm}}$. Considering the packed bed as a theoretical set of cylindrical capillaries removes the influence of the pore structure:

$$
\begin{aligned}
& I=-C_{22} \cdot \frac{A}{L} \cdot \Delta \Psi=-\frac{\Delta \Psi}{R_{\mathrm{Ohm}}}, \\
& I=-C_{22, \text { Cap }} \cdot \frac{A_{\text {Cap }}}{L_{\text {Cap }}} \cdot \Delta \Psi .
\end{aligned}
$$

If the capillary conductivity $C_{22 \text {, Cap }}$ is known, it is possible to calculate the geometry factor which relates the capillary geometry to the geometry of the packed bed as

$$
\frac{A_{\text {Cap }} \cdot L}{A \cdot L_{\text {Cap }}}=\frac{C_{22}}{C_{22, \text { Cap }}} \text {. }
$$

If surface conduction was negligible, this approach would be analogous to Archie's law. He found out that the conductivity of a porous system is a function of the liquid conductivity $K_{L}$ and the pore structure [22], but in the presence of an EDL, the capillary conductivity $C_{22 \text {,Cap }}$ also comprises surface conductivity $K_{\sigma}$ (by movement of ions in the immobile part of the EDL). $K_{\sigma}$ is related to the particle diameter $a$ to account for the specific surface area $[23,24]$

$$
C_{22, \text { Cap }}=K_{L}+4 \cdot K_{\sigma} / a .
$$

The dimensionless Dukhin number $D u$ is the ratio of surface conduction to liquid conduction $[2,23]$

$$
D u=\frac{4 \cdot K_{\sigma}}{a \cdot K_{L}} .
$$

Surface conduction must not be neglected for a correct interpretation of electrohydrodynamics since it causes a "short-circuiting" behind the shear plane of the EDL and thus does not contribute to the electroosmotic flow [2]. For a rigid structure, the geometry factor may be determined by filling the pores with a high electrolyte solution and measuring the total conductivity $\mathrm{C}_{22}$; if liquid conduction is much higher than surface conduction, $C_{22, \text { Cap }}$ equals approximately $K_{L}$. However, the technique is not applicable to packed beds since the increase of ionic strength influences the packed bed's structure substantially. Another approach is to determine the capillary conductivity $C_{22 \text {, Cap }}$ from the electroosmotic drag $\dot{V} / I$, that relates the electroosmotic flow to the electric current [15]. The electroosmotic drag may be expressed as the flow rate per coulomb or as the number of water molecules that are dragged per number of transported protons

$$
C_{22, \text { Cap }}=-\frac{\varepsilon_{L} \cdot \varepsilon_{0} \cdot \zeta}{\eta \cdot \dot{V} / I} .
$$

As fluid flow and charge transport in porous structures are closely interrelated, it is difficult to examine them separately. Especially, the charge transport mechanism has a strong influence on the electrohydrodynamic transport. The separation between surface and liquid conduction permits to access the electroosmotic flow and the streaming current with a capillary model. 


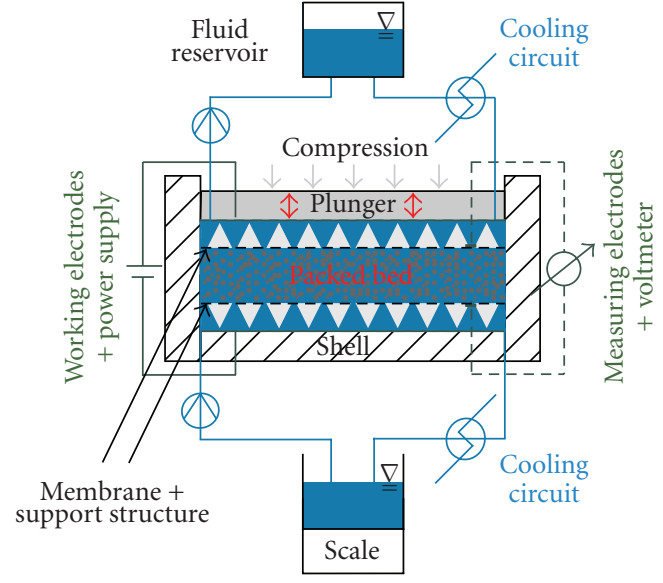

FIgURE 1: Electro-compression-permeability-cell.

\section{Experiments}

The electro-compression-permeability-cell (ECP-Cell) (see Figure 1) is designed for the compression and permeation of packed beds. These consist of ceramic particles compressed between two membranes by applying a force to the plunger. The two-sided filtration on a cross-section area of $0.005 \mathrm{~m}^{2}$ provides an even compression and thus homogeneous structure of the packed bed. After reaching a constant thickness of approximately $3 \mathrm{~mm}$ to $8 \mathrm{~mm}$ (as measured with a position encoder at the piston), the packed bed is cooled to $288 \mathrm{~K}$ by circulating electrolyte solution through the heat exchangers. The solution has the same ionic strength and $\mathrm{pH}$ value as the suspension from which the packed bed is formed.

Applying pressures of 20 and $40 \mathrm{kPa}$ to the liquid in the fluid reservoir, the flow rate and the streaming potential are measured with the scale and the measuring electrodes. These point electrodes, made from platinum, are directly in contact with the membranes. They are suitable to measure a voltage, but not an electric current. The voltmeter has an electric resistance of $10 \mathrm{M} \Omega$.

Subsequently, an electric voltage is applied to the largearea working electrodes to drive electric currents of 10, 20, 50 , and $100 \mathrm{~mA}$. The electrodes consist of titanium that is coated with iridium oxide to prevent oxidation. Reversing the electric field every 30 seconds prevents the formation of electrolytic gas; the electrodes take up hydrogen during the cathode cycle and release it during the anode cycle. Electrolytic gas would be seen on the scale as an apparent mass flow, since the gas would displace the water from the cooling circuit. The driving electric field is measured with the measuring electrodes because the electric resistance of the liquid in the cooling circuit reduces the effective voltage. The electric current is measured at the working electrodes. All parts of the ECP-Cell except for the electrodes are nonconducting.

For each $\mathrm{pH}$ value a new packed bed is formed. The suspensions are prepared by dispersing $25 \mathrm{~g}$ of boehmite particles (Disperal from Sasol, Hamburg, Germany) in $225 \mathrm{~g}$ potassium nitrate solution with an ionic strength of $0.01 \mathrm{M}$ and $\mathrm{pH}$ 4. At a low $\mathrm{pH}$ value the particles are easily dispersed by stirring with an agitator (F 52075 from Krups, Offenbach, Germany) at $800 \mathrm{rpm}$ for 30 minutes. During this time, the $\mathrm{pH}$ value is constantly readjusted by adding nitric acid. Subsequently, the final $\mathrm{pH}$ value is adjusted by adding $1 \mathrm{M}$ nitric acid or caustic potash while stirring with a horseshoe mixer. This mixer reaches the bottom as well as the wall of the beaker and has a helix form to evoke axial transport. A homogenous mixing and shear rate during the flocculation are critical because the flocculated suspensions are pseudoplastic. Using a propeller mixer would lead to an inhomogeneous shear stress and thus to an inhomogeneous distribution of the agglomerate size and the viscosity. The $\mathrm{pH}$ value of the suspension is constantly readjusted during 45 minutes of stirring.

Disperal was chosen for the experiments because it is easy to disperse at low $\mathrm{pH}$ values. The primary particles have a narrow-size distribution $\left(x_{0,10}=28 \mathrm{~nm}, x_{0,50}=\right.$ $38 \mathrm{~nm}$, and $x_{0,90}=56 \mathrm{~nm}$ as measured with a Nanotrac from Mircotrac Inc., Pa, USA). Boehmite has an IEP of 9.5 and the zeta potential is $+61 \mathrm{mV}$ at $\mathrm{pH} 4,+44 \mathrm{mV}$ at $\mathrm{pH} 7,+22 \mathrm{mV}$ at $\mathrm{pH} 8.5,+3 \mathrm{mV}$ at $\mathrm{pH} 9.5,-13 \mathrm{mV}$ at $\mathrm{pH} 10$, and $-29 \mathrm{mV}$ at $\mathrm{pH} 12$. Disperal is commonly used as a filler material in synthetics and as a raw material for the aluminium oxide industry. Boehmite is dissolvable in aqueous solutions, especially at high and low $\mathrm{pH}$ values. However, at temperatures below $50^{\circ} \mathrm{C}$ the dissolution is so slow that it may be neglected [25]. The nylon membranes (Ultipor from Pall, NY, USA) have a nominal pore size of $0.1 \mu \mathrm{m}$. The particles being smaller than the pores of the membrane are retarded because the pores get clogged.

\section{Results}

The gradients, the fluid flow, and the electric current are defined in the direction of the pressure-driven flow. With the upper working electrode defined as the anode, a negative electric potential difference means that the electric potential decreases along this direction. Protons move toward the lower working electrode to reduce their potential causing an electric current in the same direction.

Both porosity $\Phi$ and permeability $C_{11}$ (calculated with (3) and (4)) strongly depend on the $\mathrm{pH}$ value of the suspension (see Figure 2). The high permeability around the IEP (at a $\mathrm{pH}$ of about 9.5) is caused by the agglomeration of the particles. Above the IEP, the porosity and the permeability do not decrease because the electrostatic repulsion is too weak to desagglomerate the particles. A substantial change of porosity and permeability occurs between $\mathrm{pH} 8.5$ and $\mathrm{pH} 4$, where the electrostatic repulsion between the charged particles prevents agglomeration. An increase of the $\mathrm{pH}$ value (for example from 4 to 8.5 at 1 bar) causes a small increase of the porosity (by a factor of 1.26, but a large increase of permeability (by a factor of 7.10). This indicates a shrinking of the large pores between the agglomerates, which are accountable for the major part of the fluid transport $[7,20]$. Since the flow is laminar, the average velocity is higher in larger pores, so that they have a disproportionately high contribution to the fluid flow. 


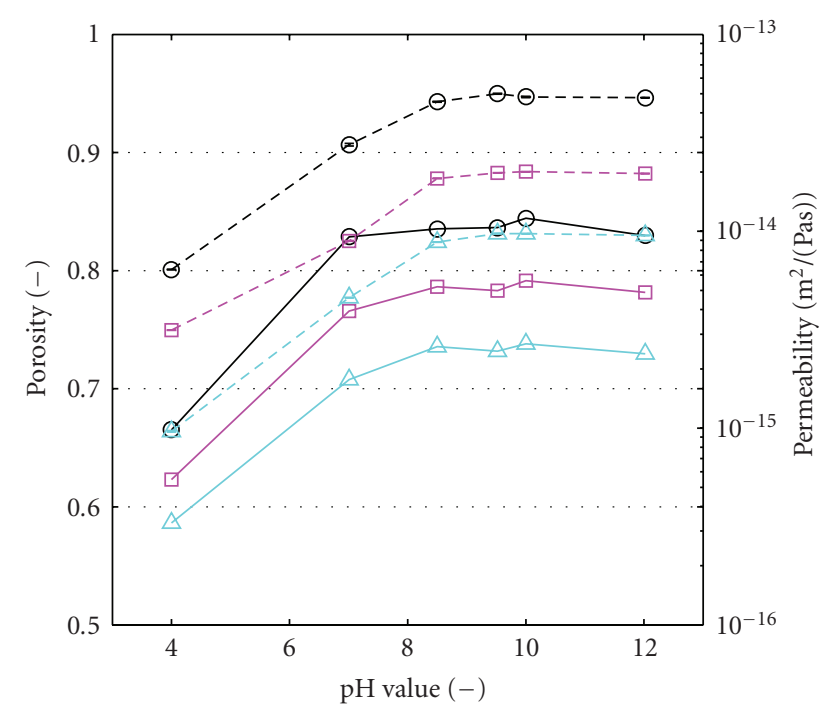

$$
\begin{aligned}
& -1 \text { bar } \\
& \square \quad 2 \text { bar } \\
& \triangle 4 \text { bar }
\end{aligned}
$$

Figure 2: Porosity $\Phi$ (full lines, left axis) and permeability $C_{11}$ (dashed lines, right axis) at different levels of compression.

Porosity $\Phi$ and permeability $C_{11}$ also decrease upon an increase of compression. The porosity change is about the same in both compression steps (from 1 bar to 2 bar and from 2 bar to 4 bar). The difference of the permeabilities at 1 bar and at 2 bar is bigger than the difference between 2 bar and 4 bar. Again, this indicates that first the largest pores between the agglomerates are narrowed, later also the smaller pores are affected.

Figure 3 shows the liquid conductivity $K_{L}$ (full lines) and the conductivity of the packed beds $C_{22}$ (dashed lines) as functions of the $\mathrm{pH}$ value. The liquid conductivity is nearly constant over a wide range of the $\mathrm{pH}$ and has a maximum at the upper end, coming from abundant oxonium ions. The conductivity of the packed bed $C_{22}$ is calculated using (14). It depends on the $\mathrm{pH}$ value and on the compression. A higher compression causes a higher volume fraction of the nonconducting particles and thus a lower conductivity. This also explains the low conductivity at low $\mathrm{pH}$ values. Another reason for the packed bed conductivity deviating from the liquid conductivity is the strong contribution of surface conductivity, which is indicated by the high Dukhin number (see Figure 5).

The electroosmotic drag $\dot{V} / I$, that is the quotient of the electroosmotic flow rate to the driving electric current, is negative below the IEP and positive above (see Figure 4, left axis, full lines). The quotients have minima at a $\mathrm{pH}$ of around 7, which can be explained with the relatively high zeta potential and relatively low Dukhin number (see Figure 5), which means that surface conduction is low. Surface conduction does not drag water molecules along and thus does not evoke electroosmotic flow.

The geometry factor relates $A_{\text {Cap }} / L_{\text {Cap }}$ to $A / L$ (see Figure 4, right axis, dashed lines). It is calculated with

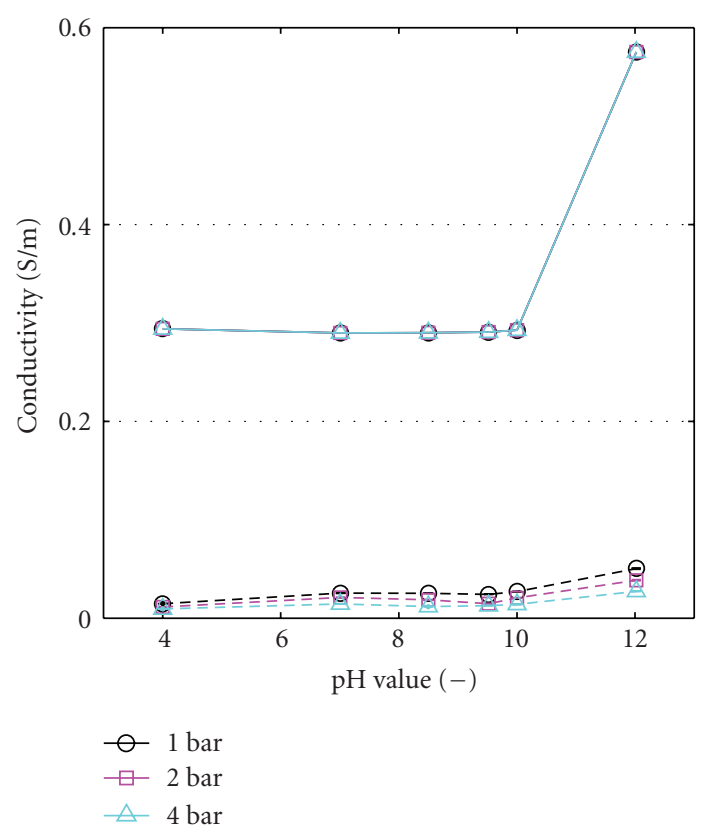

FIGURE 3: Liquid conductivity $K_{L}$ (full lines) and conductivity of the packed beds $C_{22}$ (dashed lines) at different levels of compression. The liquid conductivity does not change with compression.

(16) and (19) using the measured electroosmotic drag and the zeta potential of the particles as measured with the AcoustoSizer II. The geometry factor is smaller than 1 for all experiments because the path through the packed bed is tortuous and thus makes the capillaries longer and thinner than the packed bed. An increased compression makes the pores even more tortuous and thus reduces the geometry factor. The geometry factor has a maximum at the IEP, where the particles are strongly agglomerated.

Figure 5 shows the capillary conductivity $C_{22 \text {,Cap }}$ and the Dukhin number $\mathrm{Du}$ depending on the $\mathrm{pH}$ value. The capillary conductivity has a minimum around the IEP, because of the zero particle charge and the low liquid conductivity. The Dukhin number correlates to the capillary conductivity in the medium $\mathrm{pH}$ range. The deviation at high $\mathrm{pH}$ values is caused by the high liquid conductivity. An increased compression leads to higher capillary conductivity $C_{22 \text {,Cap }}$ and Dukhin number. Surface conduction is favored by a closer contact between the particles and thus stronger overlap of the EDL. This is not contradicted by the decreasing conductivity of the packed bed $C_{22}$ upon an increase of compression (see Figure 3 ). While the surface conductivity and thus the capillary conductivity increase; this effect is dominated by the decreasing geometry factor. The relatively high Dukhin number $D u$ is the reason for the minima of the electroosmotic drag at $\mathrm{pH} 7$ (see Figure 4, left axis, full lines). Charge transport behind the shear plane of the EDL does not drag water molecules, so it does not contribute to the electroosmotic flow.

Figure 6 (full lines) shows the electroosmosis coefficient $C_{12}$ as calculated with (5), (6), and (7) and with the geometry factor. It correlates well with the measurements (Figure 6, dashed lines). Below the IEP the surface charge is positive, 


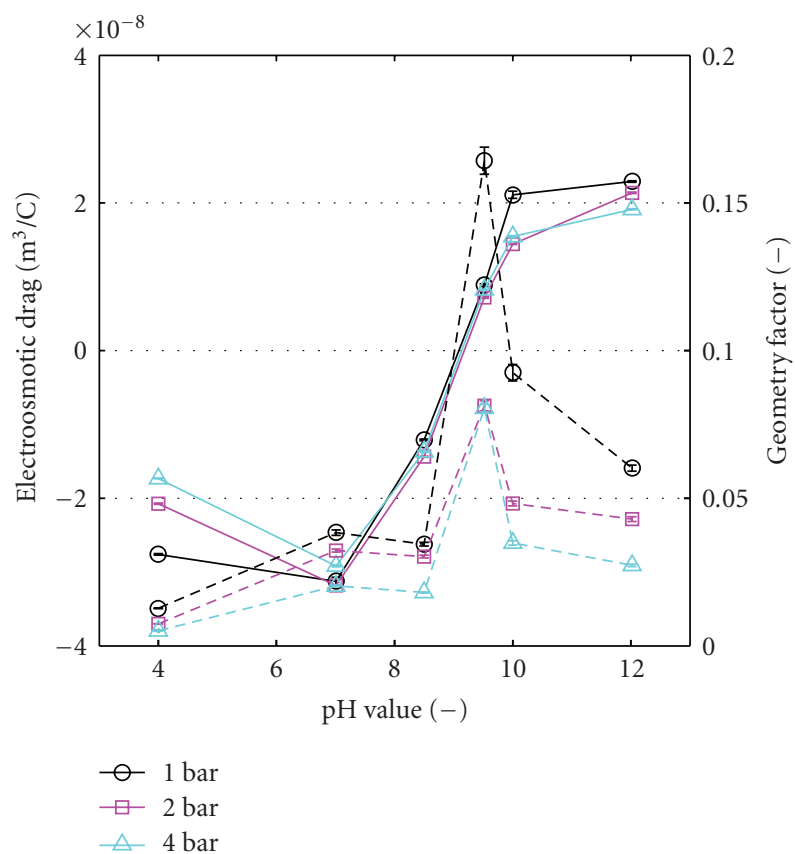

Figure 4: Electroosmotic drag $\dot{V} / I$ (full lines, left axis) and geometry factor $\left(A_{\text {Cap }} \cdot L\right) /\left(A \cdot L_{\text {Cap }}\right)$ (dashed lines, right axis) at different levels of compression.

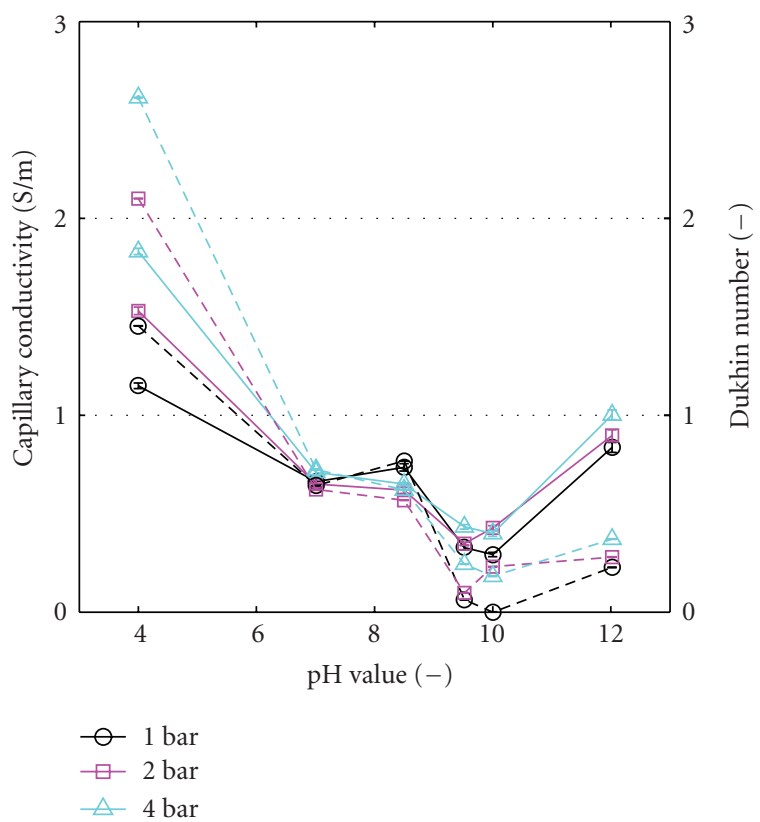

Figure 5: Capillary conductivity $C_{22, \text { Cap }}$ (full lines, left axis) and Dukhin number Du (dashed lines, right axis) at different levels of compression.

so that the counter ions in the diffuse layer are negative. If an electric field in the positive direction is applied, the negative counter ions are accelerated toward the positive electrode, which is at the upper side of the packed bed. The resulting flow is consequently negative. Accordingly, $C_{12}$ is negative below the IEP and positive above. The

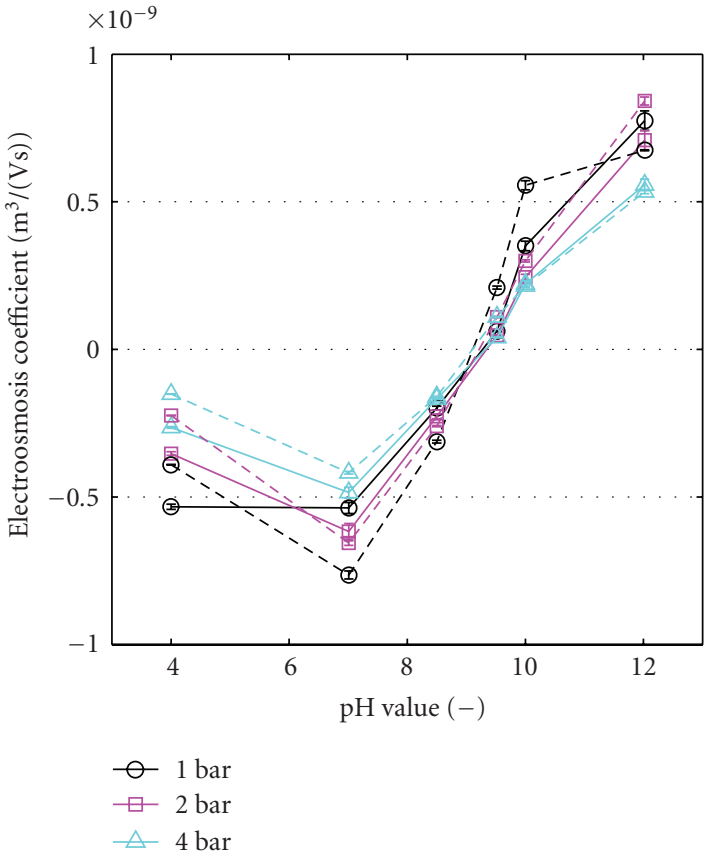

FIgURE 6: Electroosmosis coefficient $C_{12}$ as calculated (full lines) and measured (dashed lines) at different levels of compression.

electroosmotic flow is stronger at a low compression, as can be explained with the higher geometry factor and an identical $C_{12, \text { Cap }}$ in (6). The minima at $\mathrm{pH} 7$ can be explained by the relatively high zeta potential and the relatively high geometry factor. Due to Onsager's relation, the streaming current coefficient $C_{21}$ equals the electroosmosis coefficient $C_{12}$, but no experimental values exist for $C_{21}$ since the streaming current cannot be measured with the point electrodes in the ECP-Cell.

Knowing the capillary conductivity $C_{22 \text {,Cap }}$, the quotient of the streaming potential and the pressure gradient $\Delta \Psi / \Delta p$ is calculated from (11) (see Figure 7, full lines). The quotient is positive below the IEP and negative above, which is opposite to the electroosmosis coefficient $C_{12}$. At low $\mathrm{pH}$ values, the counter ions sheared off by the flow (negative pressure gradient) have a negative surface charge, so negative ions will accumulate at the downstream side, resulting in a negative electric gradient. A stronger compression leads to a smaller modulus of the quotient because of the characteristic functions of $C_{12}$ and $C_{22}$. This is also why the modulus of the quotient is smaller above the IEP than below. The calculated values agree well with the measurements (Figure 7, dashed lines). One has to consider the experimental errors of the streaming potential measurements caused by concentration polarization of the electrodes.

The electroviscosity ratio $\eta^{*} / \eta$ has a minimum at the IEP, where it is close to the theoretical value of 1 , meaning that the apparent viscosity equals the real viscosity of the liquid in the pores (see Figure 8). An increase of the surface charge leads to an increase of the electroviscosity ratio because the pressure driven flow is retarded by the electroosmotic counter flow driven by the streaming potential. Above the 


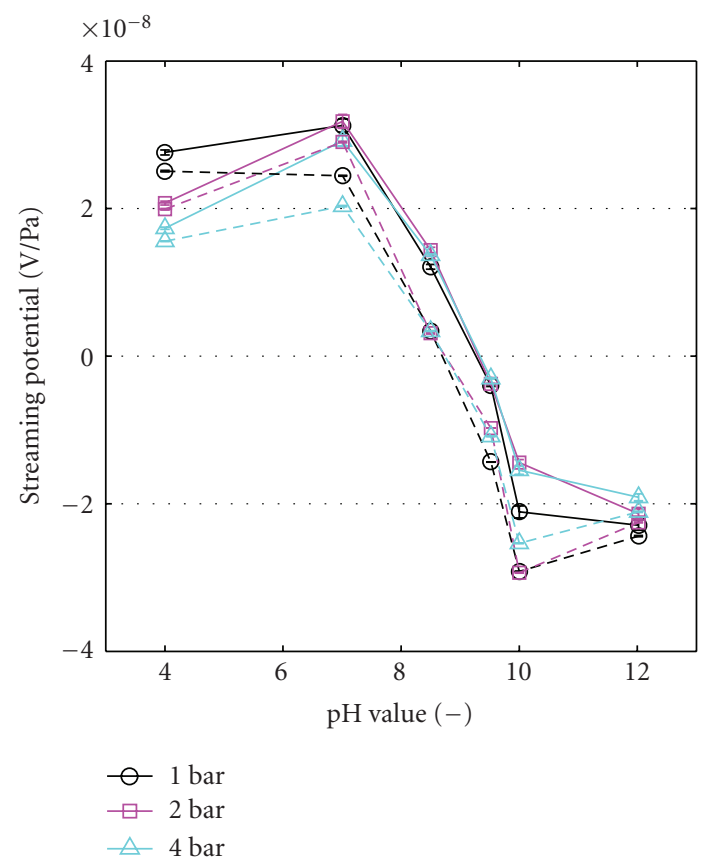

FIGURE 7: Streaming potential $\Delta \Psi$ related to pressure difference $\Delta p$ as calculated (full lines) and measured (dashed lines) at different levels of compression.

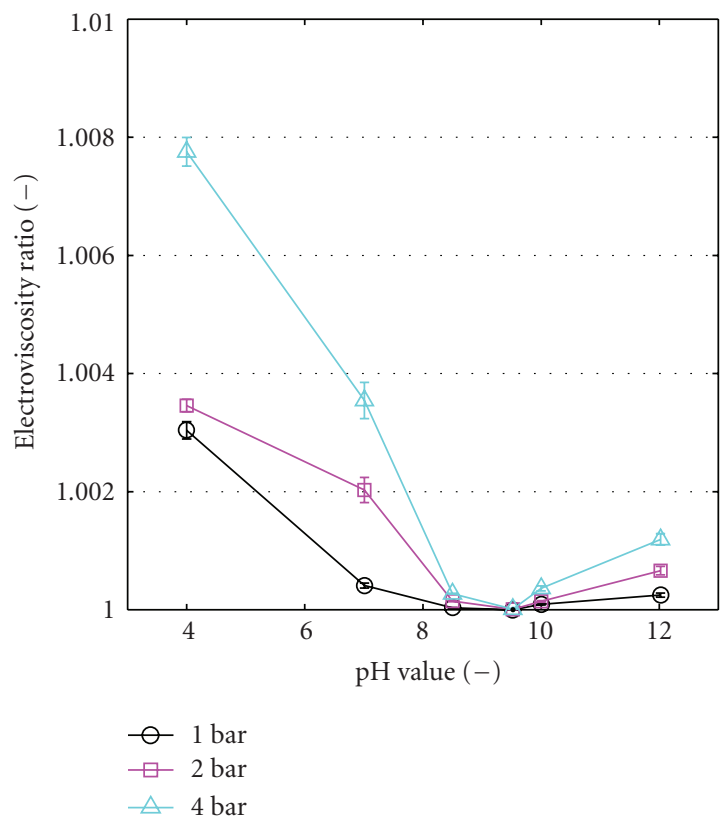

Figure 8: Electroviscosity ratio $\eta^{*} / \eta$ for different levels of compression. The error bars represent the $95 \%$ confidence interval.

IEP, the electroviscosity ratio is smaller than below because of the increased porosity. A higher porosity leads to larger pores and a smaller part of the flow taking place within the EDL. Consequently, the electroviscosity ratio increases with increasing compression. Since the increased apparent viscosity would have to be considered for the calculation of the permeability $K_{\text {Darcy }}$ we use $C_{11}$ instead of $K_{\text {Darcy }}$.

\section{Summary}

The EHT in nanoporous packed beds depends on the surface charge of the particles as well as on the pore structure, which itself depends on the surface charge. A capillary model is introduced to separate the two influences, using an experimentally determined geometry factor. The calculated charge transport and electrically driven fluid flow agree well with experimental results. The model explains why the EHT coefficients can decrease with increasing modulus of surface charge if the porosity decreases substantially.

The hydraulic permeability, however, is better described as a nonlinear relation to the porosity. The direct influence of the $\mathrm{pH}$ value on the hydraulic permeability is in the range of a few percent.

The present study is limited to the range of Smoluchowski's thin double layer approximation, which is fulfilled in many technical applications of packed beds. Here, the focus is on the influence of the $\mathrm{pH}$ value and the compression. The effect of changing the ionic strength and the particle size will be addressed in the future.

\section{Acknowledgment}

The authors would like to thank the German Science Foundation (DFG) for funding within the priority program SPP 1164.

\section{References}

[1] B. Schäfer and H. Nirschl, "Physicochemical influences on electrohydrodynamic transport in compressible packed beds of colloidal boehmite particles," Journal of Colloid and Interface Science, vol. 318, no. 2, pp. 457-462, 2008.

[2] A. V. Delgado, F. González-Caballero, R. J. Hunter, L. K. Koopal, and J. Lyklema, "Measurement and interpretation of electrokinetic phenomena," Journal of Colloid and Interface Science, vol. 309, no. 2, pp. 194-224, 2007.

[3] A. T. Yeung and J. K. Mitchell, "Coupled fluid, electrical and chemical flows in soil," Geotechnique, vol. 43, no. 1, pp. 121134, 1993.

[4] S. R. Santiwong, J. Guan, and T. D. Waite, "Effect of ionic strength and $\mathrm{pH}$ on hydraulic properties and structure of accumulating solid assemblages during microfiltration of montmorillonite suspensions," Journal of Colloid and Interface Science, vol. 317, no. 1, pp. 214-227, 2008.

[5] E. K. Zholkovskij, J. H. Masliyah, V. N. Shilov, and S. Bhattacharjee, "Electrokinetic Phenomena in concentrated disperse systems: general problem formulation and Spherical Cell Approach," Advances in Colloid and Interface Science, vol. 134-135, pp. 279-321, 2007.

[6] A. Gupta, D. Coelho, and P. M. Adler, "Universal electroosmosis formulae for porous media," Journal of Colloid and Interface Science, vol. 319, no. 2, pp. 549-554, 2008.

[7] V. Marry, J. F. Dufreche, M. Jardat, G. Meriguet, P. Turq, and F. Grun, "Dynamics and transport in charged porous media," Colloids and Surfaces A, vol. 222, no. 1-3, pp. 147-153, 2003.

[8] H. Nirschl, R. Wengeler, and B. Schäfer, "Nanofluidics in filtration and particle processing," Filtration, vol. 7, no. 1, pp. 67-74, 2007. 
[9] B. Schäfer and H. Nirschl, "Distinction between electrostatic and electroviscous effects on the permeability of colloidal packed beds," Chemical Engineering and Technology, vol. 28, no. 8, pp. 862-866, 2005.

[10] M. Wang and S. Chen, "Electroosmosis in homogeneously charged micro- and nanoscale random porous media," Journal of Colloid and Interface Science, vol. 314, no. 1, pp. 264-273, 2007.

[11] Y. Kang, S. C. Tan, C. Yang, and X. Huang, "Electrokinetic pumping using packed microcapillary," Sensors and Actuators A, vol. 133, no. 2, pp. 375-382, 2007.

[12] S. Yao, A. M. Myers, J. D. Posner, K. A. Rose, and J. G. Santiago, "Electroosmotic pumps fabricated from porous silicon membranes," Journal of Microelectromechanical Systems, vol. 15, no. 3, pp. 717-728, 2006.

[13] B. S. Pivovar, "An overview of electro-osmosis in fuel cell polymer electrolytes," Polymer, vol. 47, no. 11, pp. 4194-4202, 2006.

[14] F. G. B. Ooms, E. M. Kelder, J. Schoonman, N. Gerrits, J. Smedinga, and G. Calis, "Performance of Solupor ${ }^{\circledR}$ separator materials in lithium ion batteries," Journal of Power Sources, vol. 97-98, pp. 598-601, 2001.

[15] J. Lyklema, "Electrokinetics after Smoluchowski," Colloids and Surfaces A, vol. 222, no. 1-3, pp. 5-14, 2003.

[16] D. Antelmi, B. Cabane, M. Meireles, and P. Aimar, "Cake collapse in pressure filtration," Langmuir, vol. 17, no. 22, pp. 7137-7144, 2001.

[17] F. M. Tiller, C. S. Yeh, and W. F. Leu, "Compressibility of paniculate structures in relation to thickening, filtration, and expression-a review," Separation Science and Technology, vol. 22, no. 2-3, pp. 1037-1063, 1987.

[18] H. Darcy, Les Fontaines Publiques de la Ville de Dijon, Dalmont, Paris, France, 1856.

[19] P. C. Carman, "Fluid flow through granular beds," Transactions of the Institution of Chemical Engineers, no. 15, pp. 150166, 1937.

[20] H. Rumpf and A. R. Gupte, "Einflüsse der porosität und korngrößenverteilung im widerstandsgesetz der porenströmung," Chemie Ingenieur Technik, vol. 43, no. 6, pp. 367375, 1971.

[21] I. I. Bogdanov, V. V. Mourzenko, J.-F. Thovert, and P. M. Adler, "Effective permeability of fractured porous media with powerlaw distribution of fracture sizes," Physical Review E, vol. 76, no. 3, Article ID 036309, 15 pages, 2007.

[22] G. E. Archie, "The electrical resistivity log as an aid in determining some reservoir characteristics," Journal of Petroleum Technology, vol. 5, pp. 54-62, 1942.

[23] J. Lyklema, Ed., Fundamentals of Interface and Colloid Science II, Academic Press, London, UK, 1995.

[24] R. J. Hunter, Introduction to Modern Colloid Science, Oxford University Press, Oxford, UK, 1993.

[25] D. Panias, P. Asimidis, and I. Paspaliaris, "Solubility of boehmite in concentrated sodium hydroxide solutions: model development and assessment," Hydrometallurgy, vol. 59, no. 1, pp. 15-29, 2001. 